\title{
Systematic review and meta-analysis of the protective effect of resveratrol on multiple organ injury induced by sepsis in animal models
}

\author{
JIAWEI ZHOU ${ }^{1}$, DAIHONG YANG ${ }^{1}$, KAI LIU $^{1}$, LINYI HOU $^{2}$ and WENKAI ZHANG ${ }^{2}$ \\ ${ }^{1}$ Department of Cardiothoracic Surgery; ${ }^{2}$ Intensive Care Unit, \\ The Second Hospital of Shanxi Medical University, Taiyuan, Shanxi 030001, P.R. China
}

Received August 17, 2018; Accepted November 11, 2018

DOI: $10.3892 /$ br.2018.1169

\begin{abstract}
Sepsis may directly lead to multiple organ failure, which is among the leading causes of mortality in critically ill patients. According to data released by the Global Sepsis Alliance, the number of mortalities due to sepsis exceeded the combined number for prostate cancer, breast cancer and AIDS in 2012. To date, studies have reported that resveratrol has marked positive effects including anti-inflammatory, anti-oxidative and pro-microcirculatory functions in sepsis-induced organ injury, significantly improving the survival time and mortality of sepsis animals. The present systematic review sought to further clarify the efficacy and safety of resveratrol in the treatment of sepsis. Studies on resveratrol application in the treatment of sepsis-induced organ injury in animal models were reviewed by searching various Chinese and other language databases (PubMed, Embase, CNKI, WanFang and WeiPu) and by manually searching the references of related articles. The selection and evaluation of the studies was performed by two independent reviewers. A total of 260 related studies were initially identified. Following application of the exclusion factors and inclusion criteria, 11 studies were included. Meta-analysis revealed that resveratrol exerted significant protective effect in sepsis-induced animal models of organ injury, through anti-inflammatory, anti-oxidant and pro-microcirculatory functions compared with in the placebo group. While nuclear factor $\kappa \mathrm{B}(\mathrm{NF}-\kappa \mathrm{B})$ and nuclear factor E2-related factor $2(\mathrm{NRF}-2)$ are the two major signaling pathways to have been associated with the anti-inflammatory and anti-oxidative effects of resveratrol, these factors were not quantified for mean values, therefore
\end{abstract}

Correspondence to: Mr. Wenkai Zhang, Intensive Care Unit, The Second Hospital of Shanxi Medical University, 382 Wuyi Road, Taiyuan, Shanxi 030001, P.R. China

E-mail: 13994206729@163.com

Key words: sepsis, resveratrol, inflammatory response, oxidative stress, systematic review not suitable for systematic evaluation. For related factors, the results of meta-analysis were as follows: For tumor necrosis factor- $\alpha$ (TNF- $\alpha$ ), the standardized mean difference (SMD) was -13.50 [95\% confidence interval (CI): -22.08, -4.91; $\mathrm{P}=0.002]$; for malondialdehyde (MDA), the SMD was -3.10 (95\% CI: $-5.27,-0.93 ; \mathrm{P}=0.005$ ); for mean arterial pressure the SMD was 1.34 (95\% CI: 0.07, 2.62; $\mathrm{P}=0.04)$; for interleukin (IL)-6 the SMD was -9.57 (95\% CI: $-20.90,1.75$; $\mathrm{P}=0.10)$; and for IL-10 the SMD was 0.80 (95\% CI: $-0.73,2.34$; $\mathrm{P}=0.31)$. It was concluded that resveratrol exerted significant anti-inflammatory and anti-oxidative effects through NF- $\kappa \mathrm{B}$ and NRF-2 signaling pathways in animal models of sepsis-induced multiple organ injury, manifesting as significant downregulation of TNF- $\alpha$ and MDA expression and improved microcirculation, therefore ameliorating septic damage to the body, which may ultimately improve survival ratios.

\section{Introduction}

Sepsis is a systemic inflammatory response caused by infection with pathogens, primarily gram-negative bacteria infections as well as other bacterial, fungal and virus infections (1). The pathogenesis of sepsis involves an inflammatory response, oxidative stress, apoptosis, ischemia-reperfusion injury, endothelial dysfunction and effects mediated by endothelin (ET) peptides (2-7). Infection-induced systemic inflammatory response syndrome (SIRS), which if not treated timely, may develop into multiple organ dysfunction syndrome (MODS) $(8,9)$. Despite the widespread use of measures including antibiotics, glucocorticoids and organ function support therapy, these treatments have not significantly improved the prognosis of sepsis, and the mortality rate remains as high as $30-40 \%(10,11)$. In fact, the rates of morbidity and mortality were reported to increase yearly within three decades starting in the 1990s (12). It has been reported that at least 750,000 septic shocks occur in the United States each year, and that more than 210,000 sepsis-related mortalities occur, which is associated with annual overall losses of $\$ 16$ billion in overall economic costs associated with medicine and healthcare $(13,14)$. Thus, sepsis represents a notable burden to health. Finding effective treatments for sepsis, shortening the length of hospital stay, reducing the 
incidence of severe sepsis, reducing mortality, and reducing hospitalization costs have become a worldwide challenge in the field of critical care medicine.

Resveratrol is a non-flavonoid polyphenolic compound built around a quinone structure with reported anti-inflammatory, anti-oxidant and anticancer functions (15). Studies by Kolgazi et al (15) and Sebai et al (16) demonstrated that resveratrol reduced the expression of tumor necrosis factor- $\alpha$ (TNF- $\alpha$ ), malondialdehyde (MDA) and glutathione by reducing the accumulation of neutrophils in the lungs and kidneys, reducing the activity of myeloperoxidase and lactate dehydrogenase and further alleviating multiple organ damage induced by sepsis and improving the survival rate of rats and mice. Murakami et al (17) reported that resveratrol could reduce the activity of phospholipase A2 and reduce the expression of potent inflammatory factors including prostaglandin and lymphotoxin. Study has also indicated that resveratrol may alleviate sepsis-induced lung injury in rats by downregulating lung tissue high mobility group protein B1 and Toll-like receptor 4 (18).

Nuclear factor- $\mathrm{\kappa B}(\mathrm{NF}-\kappa \mathrm{B})$ and nuclear factor E2-related factor-2 (NRF-2) signaling pathways exert important functions in cells. Resveratrol has been implicated to be widely involved in the protection of various diseases of the body via effects on NF- $\kappa \mathrm{B}$ and NRF-2. For instance, it was reported that resveratrol could reduce lipopolysaccharide (LPS)-induced brain damage by downregulating NF- $\mathrm{KB}$ (19). Kim et al (20) reported that resveratrol inhibited the expression of inducible nitric oxide synthase and prostaglandin E2 by regulating NF- $\kappa \mathrm{B}$ activity, and effectively inhibited LPS-induced neurodegenerative diseases caused by microglial activation. Lei et al (21) reported that resveratrol activated the silent information regulator 1 (SIRT1) pathway, inhibiting nuclear translocation of NF- $\mathrm{kB}$ and acetylation of $\mathrm{p} 65$, and alleviating interleukin (IL)- $\beta$-induced inflammation of chondrocytes. In LPS-induced human monocyte cell line THP-1, resveratrol inhibited IL-8 secretion by blocking mitogen-activated protein kinase and NF- $\kappa B$ phosphorylation (22). In an adult mouse model of LPS-induced depression, resveratrol increased phosphorylated cyclic AMP response element binding protein/brain-derived neurotrophic factor expression in the frontal cortex and hippocampus by inhibiting NF- $\mathrm{\kappa B}$, to alleviate the depressive behavior of mice (23). Ma et al (24) reported that resveratrol exerted anti-inflammatory effects by inhibiting NF- $\mathrm{KB}$ and Janus kinase/signal transducers and activators of transcription pathways. However, Gualdoni et al (25) reported that resveratrol activated the NF- $\mathrm{\kappa B}$ pathway, upregulated TNF- $\alpha$ expression and decreased IL-10 expression, and had a pro-inflammatory effect in human peripheral blood.

NRF-2 is an activator of the antioxidant responsive element signaling pathway and is this involved in cellular antioxidant activity. In previous studies, resveratrol enhanced heme oxygenase-1 (HO-1) expression through NRF-2-mediated signal transduction, inhibiting inflammatory responses and activating antioxidant defense systems to protect rats from LPS-induced periodontal tissue damage $(26,27)$. Resveratrol may also upregulate NRF-2 to improve LPS-induced cardiac damage (28). Imamura et al (29) has observed that resveratrol may control NRF-2 expression and attenuate LPS-induced sensory neuronal dysfunction. Furthermore, Wang et al (30) showed that resveratrol relieved sepsis-induced acute lung injury via the phosphatidylinositol-3-kinase/NRF-2/HO-1 pathway in rats. Hao et al (31) observed that resveratrol could enhance the activity of NRF-2 in vitro and in vivo, increase the expression of HO-1 and glutamate cysteine ligase, reduce the release of TNF- $\alpha$, IL-1 $\beta$, macrophage inflammatory protein- $1 \alpha$ and 1-methylcyclopropene, and reduce the production of reactive oxygen species (ROS) induced by LPS, thereby reducing inflammation and oxidative stress damage. In recent years, studies have confirmed that the activity of NF- $\mathrm{KB}$ may be regulated by inositol-requiring enzyme-1 (IRE-1) located on the endoplasmic reticulum membrane (32). Taking resveratrol as soon as possible following sepsis may reduce acute kidney injury by inhibiting the inflammatory response induced by the IRE1-NF- $\kappa B$ pathway (32). More notably, data suggests that NF-kB-P65 may negatively regulate the expression and activity of NRF-2 (33), contributing to our present research focus on NF- $\mathrm{kB}$ and NRF-2 in the treatment of sepsis.

Other studies have observed that microcirculatory dysfunction served an important role in the process of organ damage caused by endotoxin shock $(34,35)$. In this context, it has been reported that resveratrol increased the perfusion pressure and blood flow of organs by improving microcirculation to relieve sepsis-induced organ damage (36). Pendurthi et al (37) identified that resveratrol significantly reduced the expression of tissue factor and improved the body's coagulation function in human endothelial cells and monocytes induced by sepsis.

However, the role of resveratrol in sepsis-induced injury remains controversial $(25,38)$. Therefore, the present systematic review sought to further clarify the efficacy and safety of resveratrol in the treatment of sepsis, ultimately aiming to provide a more specific theoretical basis for the application of resveratrol in clinical trials in future.

\section{Materials and methods}

Literature search and inclusion criteria. Literatures on the protective effects of resveratrol in sepsis-induced body damage were identified through databases and manual searches. The databases used were PubMed (https://www.ncbi.nlm.nih. gov/), Embase (https://biblio.ucaldas.edu.co:2118/), CNKI (http://www.cnki.net/), Wanfang (http://www.wanfangdata. com.cn/index.html), and Weipu (http://www.cqvip.com/), each searched from database establishment to August 2018. The search terms were 'sepsis' and 'resveratrol'. Qualified articles were also obtained by searching the references of related articles. Finally, we included studies that involved at least one indicator of sepsis-induced injury in the body, namely TNF- $\alpha$, MDA and mean arterial pressure (MAP), IL-6 and IL-10. The inclusion criteria of the articles were as follows: Sepsis modelling in animals, randomized controlled trials, blinded dosing and modeling, blinded acquisition of outcome indicators and the assessment of data, samples collected to assess TNF- $\alpha$, IL- 6 and IL-10 were plasma, and samples collected to assess MDA were tissue. The exclusion criteria were: Non-randomized controlled trial, non-animal study, no specific mean, no sepsis induced organ injury, and malondialdehyde measurement not in lung tissue. The selected languages were Chinese and English. Controversial studies and data were verified by author Zhang Wenkai. 
Data extraction and methodological quality evaluation. To ensure the suitability of the included studies, all of the final inclusions were independently reviewed by two authors. The basic characteristics were determined of the articles, by reading the title, abstract and key words, and finally the full text, including the septic injury method and sex of the animals, the number of animals in the experimental and placebo groups, the method of modeling, the place and dose of administration, the site of injury, and the observed indicators. The methodological quality evaluation of included literatures was assessed on the following: Randomization of the treatment allocation, blinded drug administration, blinded outcome assessment and outcome measurements including the degree of inflammatory response (TNF- $\alpha$ level).

Analysis of data. For the obtained continuous data, the validity was estimated by calculating standardized mean difference (SMD) and 95\% confidence intervals (CIs). For the dichotomous data, odds ratios (ORs) were calculated. The RevMan 5.0 software was used for the meta-analysis and the heterogeneity of the studies was evaluated by the $\mathrm{I}^{2}$ statistic. $\mathrm{P}<0.05$ was considered to indicate statistical significance.

\section{Results}

Description of the included studies. Of the 260 studies identified by initial searches, 11 met the requirements (15,39-47,53) (Fig. 1). Among these were 4 studies in the English language and 7 in Chinese. The basic characteristics of these 11 studies were characterized, as shown in Table I.

Methodological quality evaluation of the included literature. All studies described the random assignment of experimental animals, however in all studies the specific random assignments were not stated; furthermore the blinding method was not described for animal modeling and resveratrol administration, and the data obtained and the analysis of the data did not indicate blinding. In addition, the data representing the inflammatory response condition varied greatly among studies. For certain studies, there were defects in certain aspects. For the measurement of TNF- $\alpha$, the specimens collected in one study was lung tissue rather than plasma (39). Although resveratrol could significantly reduce the expression of TNF- $\alpha$ in animal models of sepsis-induced multiple organ injury, TNF- $\alpha$ had no exact mean values in some studies (40-42), but corresponding data was displayed in a figure or table. Similarly, MDA did not have an exact mean value in one study (15). Some of the experimental animals had obesity prior to modeling (43), and some animals had albinism (41). The acquisition time of samples from one study was unclear (44). Regarding the safety of the dose of resveratrol, none of the included studies mentioned this. Furthermore, in two of the studies the number of animals included in each group was not well defined $(15,43)$. No studies described the number of animals that succumbed by chance.

\section{Data analysis}

$T N F-\alpha$. Of the 11 studies included, 8 studies measured TNF- $\alpha(15,39-42,44-46)$, and 4 studies were included in the

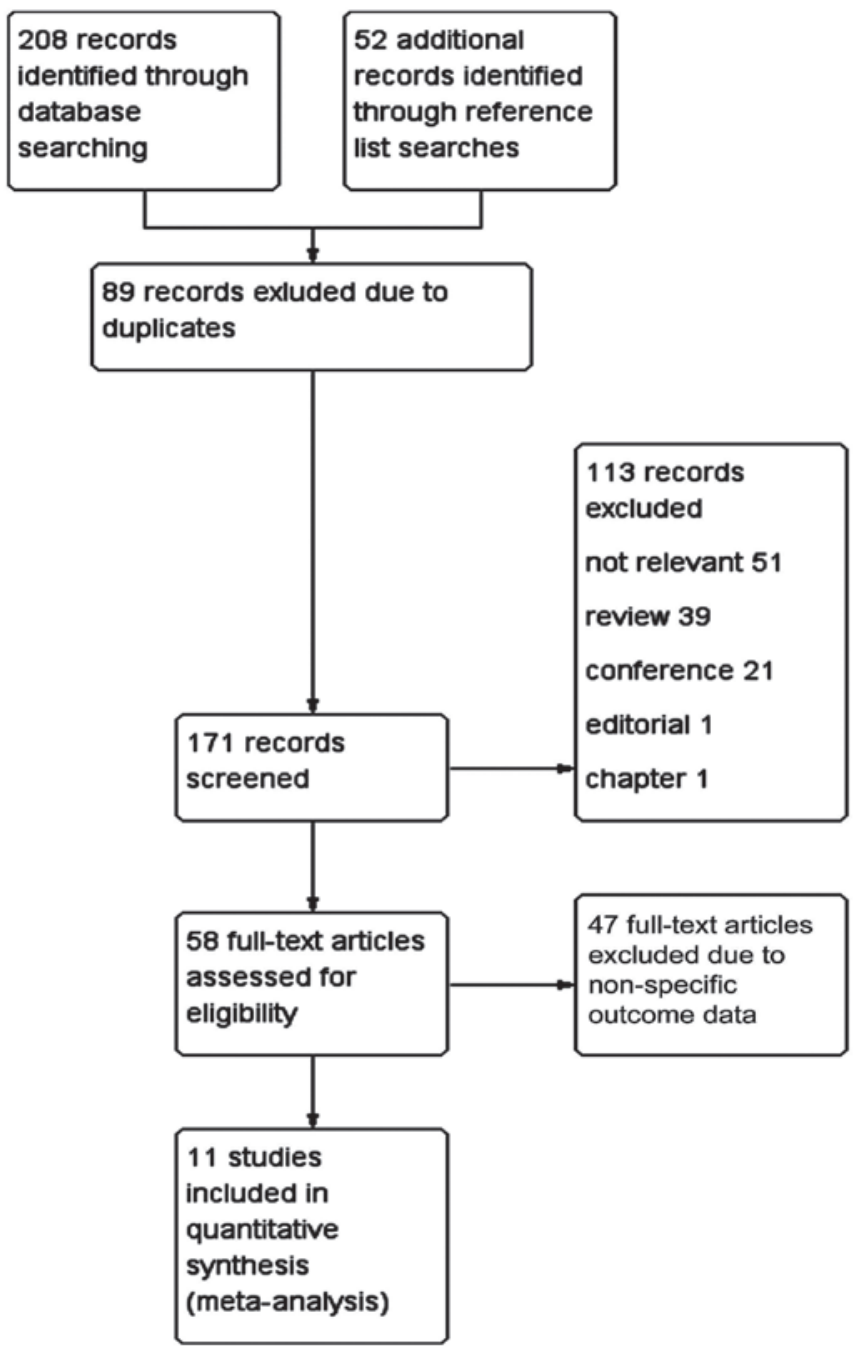

Figure 1. Flow chart of study selection process. PubMed, Embase, CNKI, Wanfang and Weipu were searched. The search terms were 'sepsis' and 'resveratrol'. A total of 208 articles were included. Another 52 articles were obtained that met the requirements by searching the references of related articles. Of the 260 articles identified, 89 were duplicated. By reading the title and abstract, another 113 articles were excluded: 51 articles were not relevant, 39 articles were a review, 21 articles were a conference, 1 was an editorial and 1 was a chapter article. There were 58 articles left. Then through review of the full text, 47 articles were found to have no specific values for the outcome indicators for meta-analysis. Finally, articles containing at least one outcome indicator were included, amounting to 11 articles.

meta-analysis (15,44-46), as shown in Fig. 2. The SMD was -13.50 (95\% CI: $-22.08,-4.91 ; \mathrm{P}=0.002)$. These results indicated that TNF- $\alpha$ was significantly reduced by resveratrol treatment, compared with in the respective model controls.

Of the 8 studies, 3 studies did not give an exact mean value (40-42). Although the other excluded study gave an exact mean, the collected specimens were lung tissue rather than plasma (39). These studies all mentioned that resveratrol could significantly reduce the expression of TNF- $\alpha$, but due to the lack of mean values they could not be used for the meta-analysis.

$M D A$. Five of the included studies measured MDA $(15,39-41,44)$, however one did not have a specific mean (15). Of the 5 studies, 2 measured MDA in multiple organs (spleen, liver, kidney, lung, small intestine, colon) $(40,41)$, 


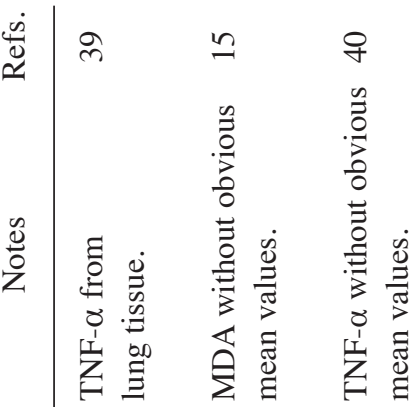

ก

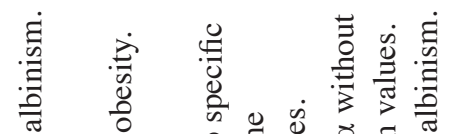

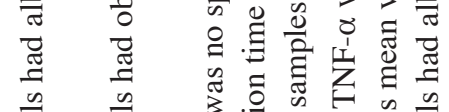

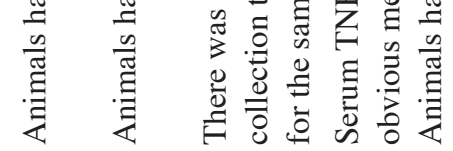

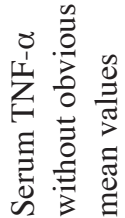

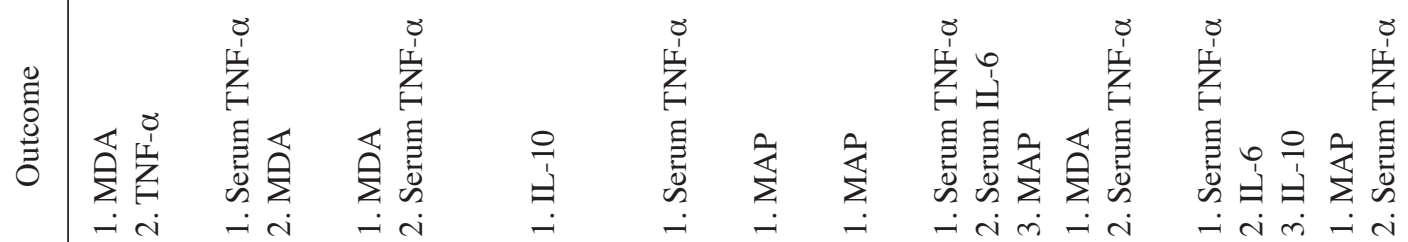

(2)

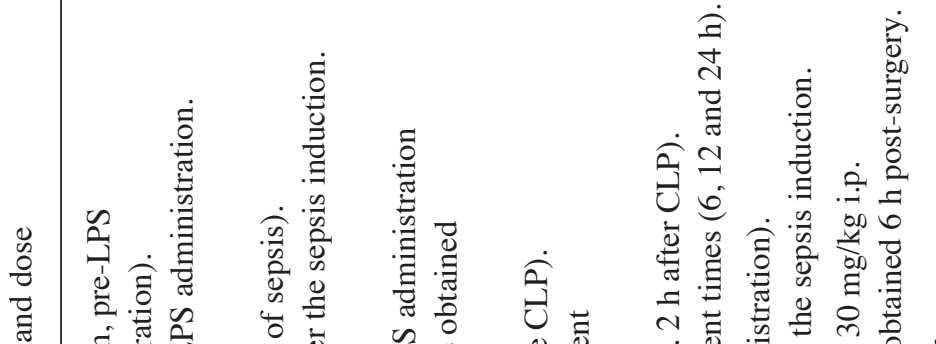

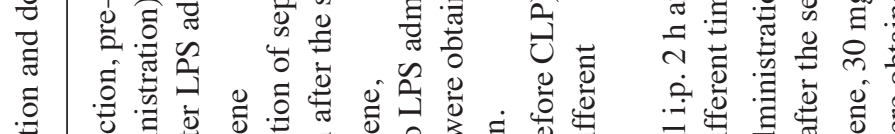

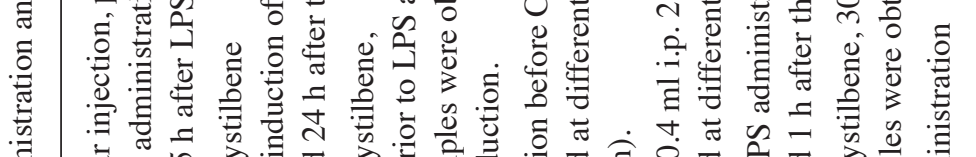

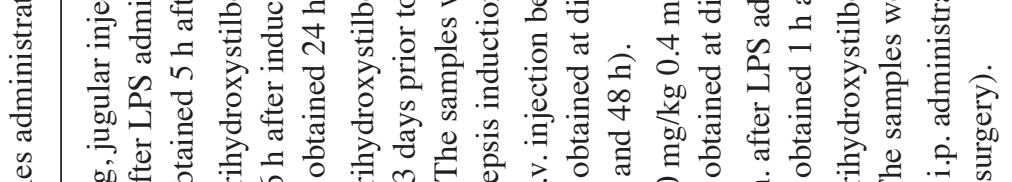

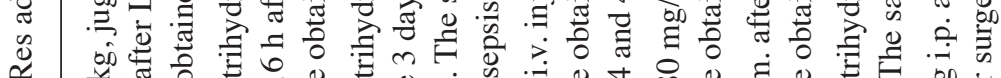

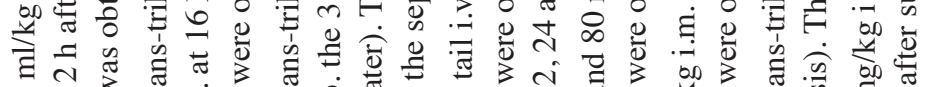

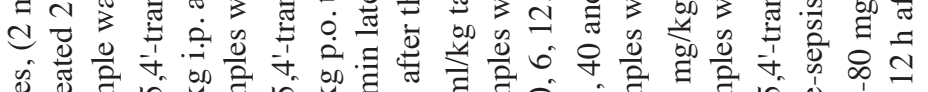

品 卷

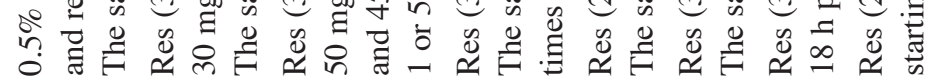

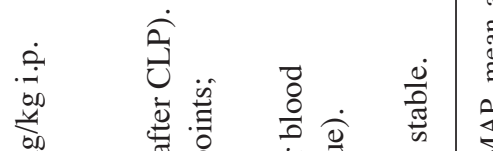

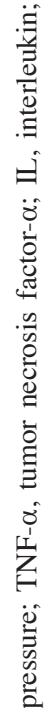

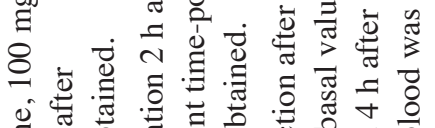

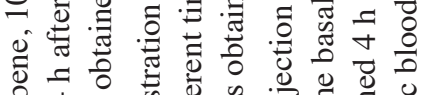

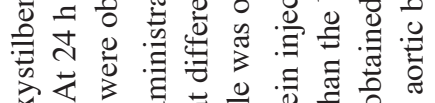

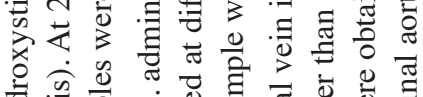

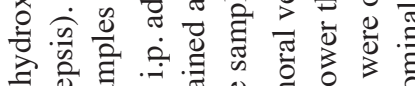

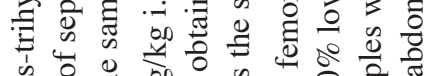

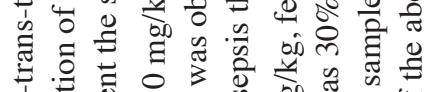

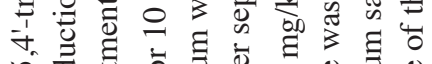

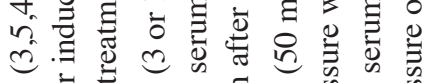

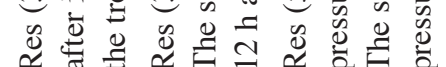

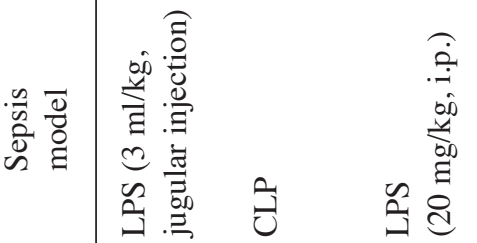

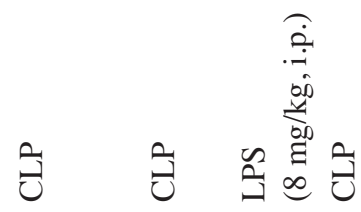

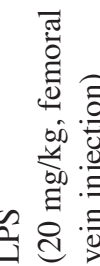

0
.0
0
0
0
0
0
0
0
0
0
0

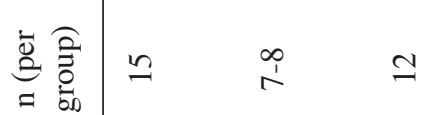

o $\infty$

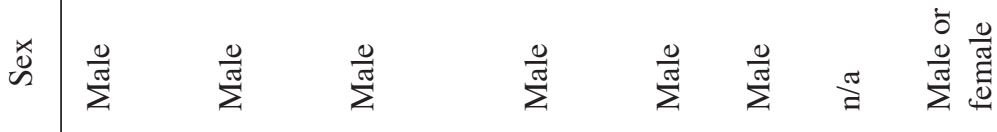

迎 造

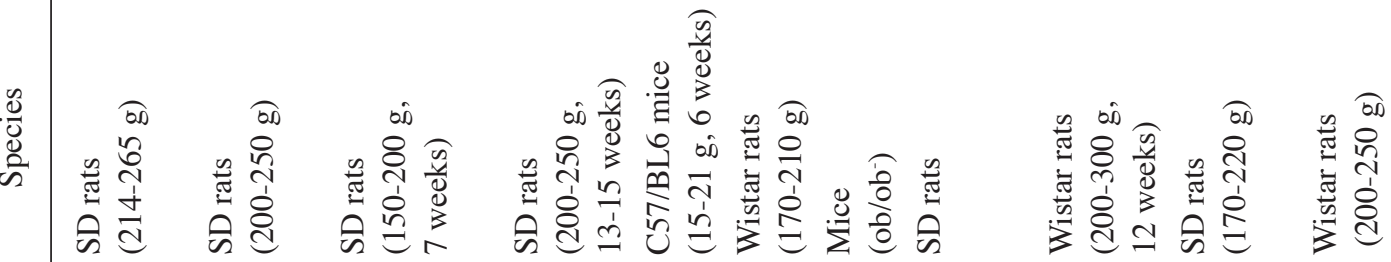

诺

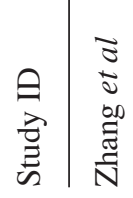
i $\overline{2}$

童 咅

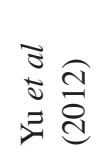

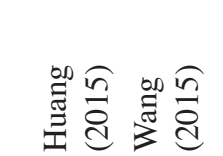

$\overline{2}$ 


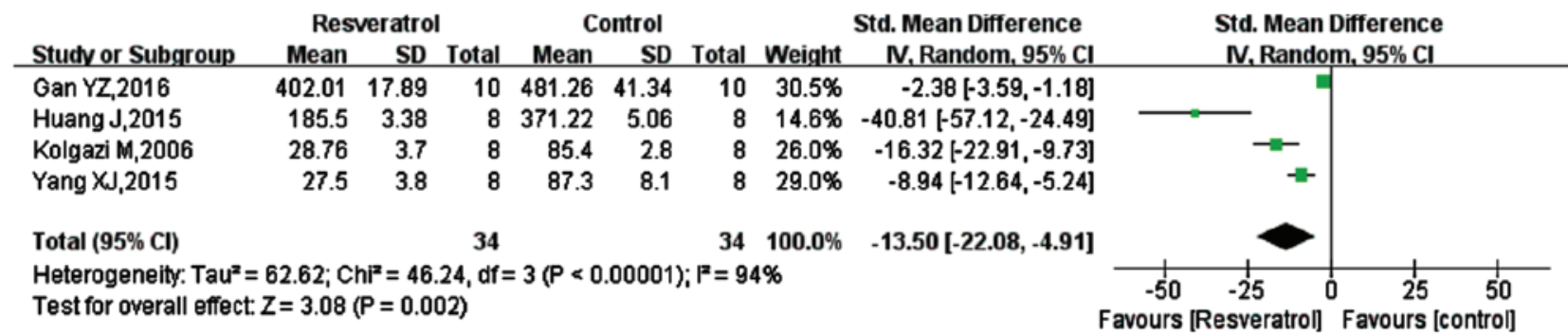

Figure 2. Comparison of the effect on TNF- $\alpha$ between resveratrol and control treatments in the plasma of animal sepsis models. The prism represents the statistical result of the overall experimental data, the squares represent the weight of each study, and the horizontal lines represent the $95 \%$ CIs for each study. The results showed that compared with control treatment, TNF- $\alpha$ was significantly reduced by resveratrol. TNF- $\alpha$, tumor necrosis factor- $\alpha$; CI, confidence interval; SD, standard deviation; IV, independent variable.

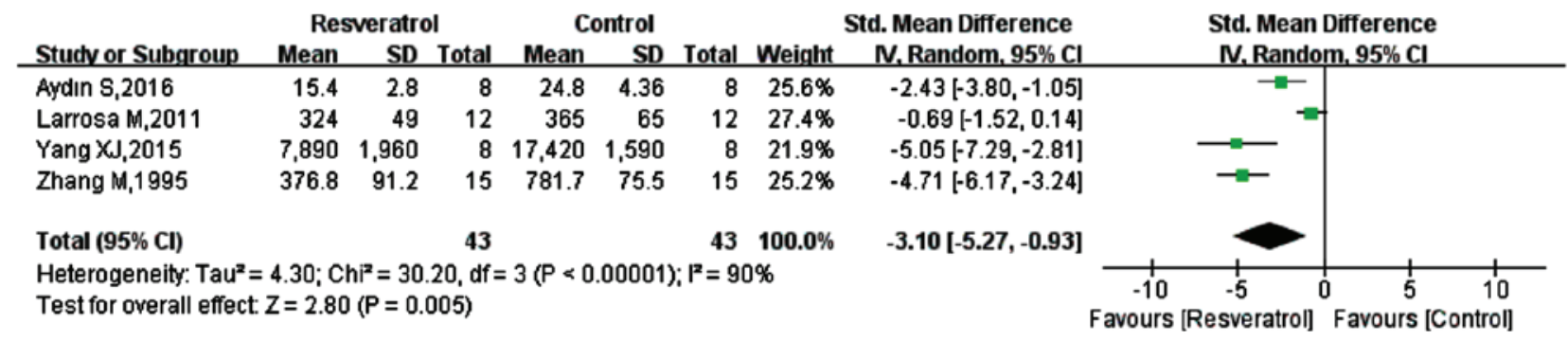

Figure 3. Comparison of the effect on MDA between resveratrol and control treatments in the tissue of animal sepsis models. The prism represents the statistical result of the overall experimental data, the squares represent the weight of each study, and the horizontal lines represent the 95\% CIs for each study. The results showed that compared with control treatment, MDA was significantly reduced by resveratrol. MDA, malondialdehyde; CI, confidence interval; SD, standard deviation; IV, independent variable.

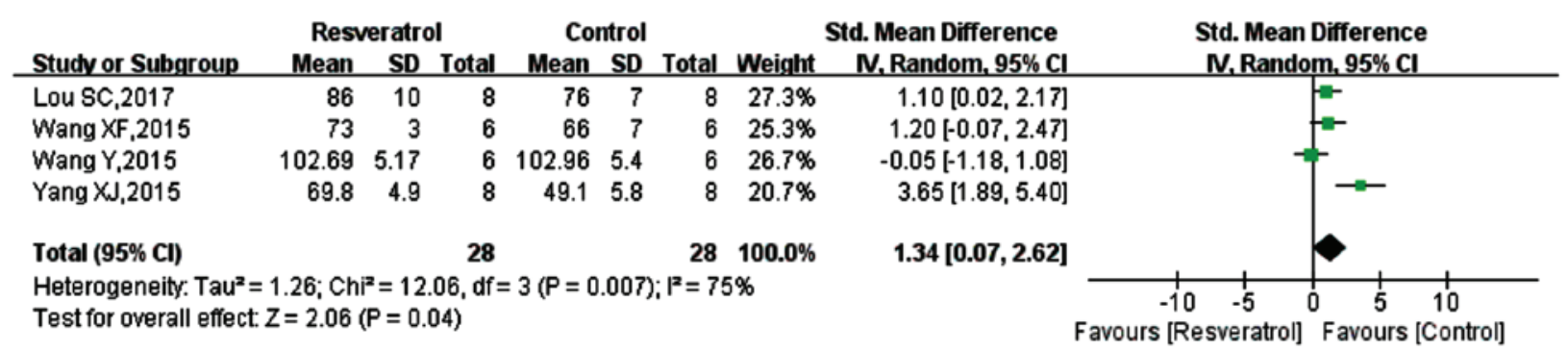

Figure 4. Comparison of the effect on MAP between resveratrol and control treatments. The prism represents the statistical result of the overall experimental data, the squares represent the weight of each study, and the horizontal lines represent the 95\% CIs for each study. The results showed that MAP was lowered following control treatment, and was increased with resveratrol application. MAP, mean arterial pressure; CI, confidence interval; SD, standard deviation; IV, independent variable.

1 measured MDA in lung and kidney (15), 1 only in the heart (44) and 1 in lung tissue (39). Analysis of the data from the 4 eligible studies is shown in Fig. 3; the SMD was -3.10 (95\% CI: $-5.27,-0.93 ; \mathrm{P}=0.005$ ). These results indicated that in contrast to the model groups, MDA was significantly reduced in the resveratrol groups.

MAP. Of the included studies, 4 studies measured MAP (42-44,47), the data from which were analyzed (Fig. 4). The SMD was 1.34 (95\% CI: $0.07,2.62 ; \mathrm{P}=0.04)$. These results suggested that MAP was reduced following sepsis modeling, while MAP was increased in models of sepsis-induction with resveratrol application. However, previous data has indicated that resveratrol does not significantly improve blood pressure or heart rate in sepsis-induced animal models (36), though was not included in the current systemic review due to the lack of accurate values.
IL-6. Of the included studies, 2 studies measured IL-6 $(44,45)$. Meta-analysis of the data from these studies, as presented in Fig. 5, showed the SMD to be -9.57 (95\% CI: -20.90, 1.75; $\mathrm{P}=0.10$ ). Thus the overall results suggested that the effect of resveratrol on the expression of IL-6 was not statistically significant. However, the data of the two studies individually demonstrated that resveratrol could significantly reduce the expression of IL-6 in animal models induced by sepsis. There were 4 other studies that sepsis-induced animal models that were not included, in which resveratrol could significantly reduce the expression of IL-6, but the results were depicted graphically $(32,48-50)$. One of these studies also showed that resveratrol reduced the expression of IL-6 in a dose-dependent manner (48). As these studies did not gave accurate means they were excluded.

$I L-10$. IL-10 is an anti-inflammatory factor involved in the body's injury response to sepsis (51-52). A total of 6 studies 


\begin{tabular}{|c|c|c|c|c|c|c|c|c|c|}
\hline \multirow[b]{2}{*}{ Stucty or Subgroup } & \multicolumn{2}{|c|}{ Resveratrol } & \multicolumn{3}{|c|}{ Control } & \multicolumn{3}{|r|}{ Std. Mean Difference } & \multirow{2}{*}{$\begin{array}{l}\text { Std. Mean Difference } \\
\text { N. Random. } 95 \% \mathrm{Cl}\end{array}$} \\
\hline & Mean & SD & Total & Mean & SD & Total & Weight & N. Random, $95 \% \mathrm{Cl}$ & \\
\hline Gan YZ,2016 & $1,392.93$ & 142.51 & 10 & $1,937.56$ & 102.43 & 10 & $53.7 \%$ & $-4.20[-5.90,-2.51]$ & \\
\hline Yang XJ,2015 & 54.7 & 6.9 & 8 & 143.1 & 2.9 & 8 & $46.3 \%$ & $-15.79[-22.17,-9.41]$ & \\
\hline Total (95\% Cl) & & & 18 & & & 18 & $100.0 \%$ & $-9.57[-20.90,1.75]$ & \\
\hline $\begin{array}{l}\text { Heterogeneity: Tau } \\
\text { Test for overall effec }\end{array}$ & $\begin{array}{l}61.48 ; \mathrm{Chi} \\
\mathrm{Z}=1.66(\mathrm{P}\end{array}$ & $\begin{array}{l}=11.84 \\
=0.10)\end{array}$ & $d f=1$ & $(\mathrm{P}=0.000$ & $6) ; P^{2}=9$ & & & & $\begin{array}{ccccc} & 1 & 1 & 1 \\
-100 & -50 & 0 & 50 & 100 \\
\text { Favours [Resveratrol] } & \text { Favours [Control] }\end{array}$ \\
\hline
\end{tabular}

Figure 5. Comparison of the effect on IL-6 between resveratrol and control treatments in the plasma of animal sepsis models. The prism represents the statistical result of the overall experimental data, the squares represent the weight of each study, and the horizontal lines represent the $95 \%$ CIs for each study. The overall results showed that the effect of resveratrol on IL-6 expression was not statistically significant; however the data from the two articles individually showed that resveratrol could be used in sepsis-induced animal models to significantly reduce IL-6 expression. IL-6, interleukin-6; CI, confidence interval; SD, standard deviation; IV, independent variable.

\begin{tabular}{|c|c|c|c|c|c|c|c|c|c|c|}
\hline \multirow[b]{2}{*}{ Studv or Subgroup } & \multicolumn{3}{|c|}{ Resveratrol } & \multicolumn{2}{|c|}{ Control } & \multirow[b]{2}{*}{ Total } & \multicolumn{2}{|r|}{ Std. Mean Difference } & \multirow{2}{*}{\multicolumn{2}{|c|}{$\begin{array}{c}\text { Std. Mean Difference } \\
\text { N. Random. } 95 \% \mathrm{Cl}\end{array}$}} \\
\hline & Mean & SD & Iotal & Mean & SD & & Weight & N. Random, 95\% Cl & & \\
\hline Gan YZ,2016 & 1.82 & 0.17 & 6 & 1.82 & 0.17 & 6 & $48.8 \%$ & $0.00[-1.13,1.13]$ & & \\
\hline Yu Yx,2012 & 242.76 & 20.33 & 10 & 208.47 & 21.52 & 10 & $51.2 \%$ & $1.57[0.54,2.60]$ & 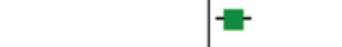 & \\
\hline Total $(95 \% \mathrm{Cl})$ & & & 16 & & & 16 & $100.0 \%$ & $0.80[-0.73,2.34]$ & & \\
\hline $\begin{array}{l}\text { Heterogeneity. Tauª } \\
\text { Test for overall effect }\end{array}$ & $\begin{array}{l}0.93 ; \mathrm{Chi} \\
\mathrm{Z}=1.02\end{array}$ & $\begin{array}{l}I^{2}=4.04 \\
(P=0.3\end{array}$ & & $=0$. & $=$ & & & & $\begin{array}{cccc}1 & 1 & 1 & 1 \\
-10 & -5 & 0 & 5 \\
\text { uurs [experimental] } & \text { Favours [co }\end{array}$ & $\left.\begin{array}{c}10 \\
\text { untrol] }\end{array}\right]$ \\
\hline
\end{tabular}

Figure 6. Comparison of the effect on IL-10 between resveratrol and control treatments in the plasma of animal sepsis models. The prism represents the statistical result of the overall experimental data, the squares represent the weight of each study, and the horizontal lines represent the 95\% CIs for each study. The overall results showed that the effect of resveratrol on IL-10 expression was not statistically significant; however the data from the two studies individually indicated that resveratrol could significantly increase the expression of IL-10. IL-10, interleukin-10; CI, confidence interval; SD, standard deviation; IV, independent variable.

measured the expression of IL-10 $(32,45,53,48-50)$, but only 2 gave mean values. Therefore, these were included in the meta-analysis $(45,53)$, as shown in Fig. 6. The SMD was 0.80 (95\% CI: $-0.73,2.34 ; \mathrm{P}=0.31$ ). Thus, the effect of resveratrol on IL-10 expression appeared to be non-significant. However, the results of the 6 studies individually suggested that resveratrol significantly increased the expression of IL-10.

\section{Discussion}

Results from the present systematic review demonstrated the protective effects of resveratrol in sepsis-induced animal model. Resveratrol may exert anti-inflammatory and anti-oxidative effects through two important signaling pathways, NF- $\mathrm{B}$ and NRF-2; however none of the initial 260 reports provided specific values for the systematic evaluation of these factors. Of these literatures, only 11 studies were deemed eligible for inclusion. Meta-analysis of these 11 studies showed that resveratrol significantly reduced the expression of TNF- $\alpha$ and MDA, and increased MAP in the sepsis animal models. However, the effect of resveratrol on downregulation of the inflammatory mediator IL- 6 and on upregulation of the anti-inflammatory mediator IL-10 did not show statistical differences. However, previous data has indicated that resveratrol does not significantly improve blood pressure or heart rate in sepsis-induced animal models (36), though was not included in the current systemic review due to the lack of accurate values. Contact with the authors was attempted to obtain more accurate values for NF- $\kappa \mathrm{B}$ NRF-2, IL-6 and IL-10; however was unsuccessful. Due to the small sample sizes in each pooled analysis, the analysis of each indicator would have had some bias.
The present article only reported on animal models with regard to the anti-inflammatory and anti-oxidative effects of resveratrol in the treatment of sepsis. However, during the literature search, it was noted that resveratrol also reduced the expression of inflammatory factors and oxidative stress products induced by sepsis at the human cell level. Cerqueira et al (54) reported that resveratrol significantly reduced the production of ROS, intercellular adhesion molecule 1 (ICAM-1), human $\beta$-defensin-2 and cell surface Fas receptor, inhibited caspase-3 and -7 activation, and upregulated glutathione peroxidase expression in a Pseudomonas aeruginosa-induced A549 cell injury model. Silswal et al (55) reported that resveratrol inhibited expression of inflammatory factors in LPS-induced human cluster of differentiation (CD) $14^{+}$monocytes, including of vascular cell adhesion molecule 1, ICAM1, C-reactive protein and resistin, and activity of proteasome subunit low-molecular-weight protein 7. Other studies have shown that resveratrol inhibited the phosphorylation of NF- $\mathrm{NB}-\mathrm{p} 65$ and the expression of inflammatory factors in human THP-1 cells following LPS induction $(37,56)$. Furthermore, it has been reported that in vitro, resveratrol served as an activator of SIRT-1, which significantly downregulated the expression of inflammatory factors, such as TNF- $\alpha$ (57). Sebai et al (58) suggested that resveratrol may participate in anti-inflammatory responses by downregulating the expression of CD14 on the cell surface, and anti-oxidation effects through the TLR4-myeloid differentiation primary response 88 or TIR-domain-containing adapter-inducing interferon- $\beta$ pathway.

Due to limited data and the lack of attention to the safety of animal experiments, few studies have reported the safety of resveratrol in animals. The quantity of resveratrol used in the studies included in the current review was within $100 \mathrm{mg} / \mathrm{kg}$, 
though the number of administrations and the route of administration differed. Two of the studies reported that as the dose of resveratrol increased, the decrease in TNF- $\alpha$ and IL- 6 , and the increase in IL-10 and MAP were more obvious $(40,44)$. There were also some differences in the timing of administration. Seven of the included studies administered resveratrol following modeling $(15,41,42,44-47)$, and two were administered prior to modeling $(43,53)$. There were two cases of administration both prior to and following modeling $(39,40)$. None of the studies assessed the effect of the time of administration on the efficacy of the drug. However, a study not included herein review reported that post-modeling administration reduced the expression of TNF- $\alpha$, IL-6, IL-1 $\beta$ and monocyte chemoattractant protein-1 to a greater extent than preoperative administration (50).

There was a degree of heterogeneity in the included studies, and the quality of the methodologies for each study was not high. The studies only illustrated the random grouping of animals and did not indicate how the experimental animals were randomly assigned. The original number of animals, the number of mortalities during the experiment, and the final remaining number were not clearly defined in any study. Furthermore, it was not stated whether the experimental data were blindly evaluated. Obviously these requirements are of key importance for human clinical research. Attempts were also made to contact the authors to obtain more raw data and detailed experimental procedures, including how animals were randomly assigned, experimental data acquisition and analysis methods, but most of the information remained unknown due to unsuccessful attempts at contact with authors. The current article was a systematic review of published studies with complete data, and studies with negative results but incomplete data were not included. Therefore, due to the existence of publication bias, results of the present systematic review are limited.

In conclusion, the present systematic review demonstrated that resveratrol does exert anti-inflammatory, anti-oxidant and pro-microcirculatory effects in sepsis-induced organ damage in animal models. However, in vivo studies of resveratrol in sepsis appear limited to animal models, and considering the quality of the studies included, the study of resveratrol in humans is still required.

\section{Acknowledgements}

The authors would like to acknowledge Dr Tao Zhou at the Department of Information and Communication Engineering, University of Electronic Science and Technology, Chengdu, China for his assistance in editing and revising the manuscript.

\section{Funding}

The present study was supported by grants from the Scientific Research Funding Project for Returnees in Shanxi Province of China (grant no. 2011-105) and the Taiyuan Science and Technology Project of Shanxi Province of China (grant no. 12016905).

\section{Availability of data and materials}

All data generated or analyzed during this study are included in this published article.

\section{Authors' contributions}

All authors were equally involved in the conception and design of the work, as well as in writing and modifying the manuscript. JZ and DY searched the databases and initially screened the included literature. WZ gave final decision of the literature to be included. LH evaluated the quality of the literatures included. KL produced the figure and tables. All authors agreed to the version to be published and to be responsible for all aspects of the work to ensure that issues related to the accuracy or completeness of the work are resolved.

\section{Ethics approval and consent to participate}

Not applicable.

\section{Patient consent for publication}

Not applicable.

\section{Competing interests}

The authors declare that they have no competing interests.

\section{References}

1. Annane D, Bellissant E and Cavaillon JM: Septic shock. Lancet 365: 63-78, 2005.

2. Greco M, Palumbo C, Sicuro F and Lobreglio G: Soluble Fms-like tyrosine kinase-1 is a marker of endothelial dysfunction during sepsis. J Clin Med Res 10: 700-706, 2018

3. Lundberg OH, Bergenzaun L, Rydén J, Rosenqvist M, Melander O and Chew MS: Adrenomedullin and endothelin-1 are associated with myocardial injury and death in septic shock patients. Crit Care 20: 178, 2016.

4. Mai N, Prifti L, Rininger A, Bazarian H and Halterman MW: Endotoxemia induces lung-brain coupling and multi-organ injury following cerebral ischemia-reperfusion. Exp Neurol 297: 82-91, 2017

5. Wan P, Tan X, Xiang Y, Tong $\mathrm{H}$ and Yu M: PI3K/AKT and CD40L signaling regulate platelet activation and endothelial cell damage in sepsis. Inflammation 41: 1815-1824, 2018.

6. Wang P, Hu Y, Yao D and Li Y: Omi/HtrA2 regulates a mitochondria-dependent apoptotic pathway in a murine model of septic encephalopathy. Cell Physiol Biochem 49 2163-2173, 2018.

7. Yamaguchi J, Nagase M, Yamamoto Y, Sakurai A, Kubo A, Mitsuhashi H, Matsuoka M, Ihara S and Kinoshita K: Increased oxidative stress and renal injury in patients with sepsis. J Clin Biochem Nutr 63: 137-143, 2018.

8. Dharap SB and Ekhande SV: An observational study of incidence, risk factors and outcome of systemic inflammatory response \& organ dysfunction following major trauma. Indian J Med Res 146: 346-353, 2017.

9. Qiao Z, Wang W, Yin L, Luo P, Greven J, Horst K and Hildebrand F: Using IL-6 concentrations in the first $24 \mathrm{~h}$ following trauma to predict immunological complications and mortality in trauma patients: A meta-analysis. Eur J Trauma Emerg Surg 44: 679-687, 2018.

10. Minamino T and Komuro I: Regeneration of the endothelium as a novel therapeutic strategy for acute lung injury. J Clin Invest 116: 2316-2319, 2006.

11. Winters BD, Eberlein M, Leung J, Needham DM, Pronovost PJ and Sevransky JE: Long-term mortality and quality of life in sepsis: A systematic review. Crit Care Med 38: 1276-1283, 2010.

12. Tsalik EL and Woods CW: Sepsis redefined: The search for surrogate markers. Int J Antimicrob Agents 34 (Suppl 4): S16-S20, 2009.

13. Wood KA and Angus DC: Pharmacoeconomic implications of new therapies in sepsis. Pharmacoeconomics 22: 895-906, 2004. 
14. O'Neill R, Morales J and Jule M: Early goal-directed therapy (EGDT) for severe sepsis/septic shock: Which components of treatment are more difficult to implement in a community-based emergency department? J Emerg Med 42: 503-510, 2012.

15. Kolgazi M, Şener G, Çetinel S, Gedik N and Alican I: Resveratrol reduces renal and lung injury caused by sepsis in rats. J Surg Res 134: 315-321, 2006

16. Sebai H, Sani M, Ghanem-Boughanmi N and Aouani E: Prevention of lipopolysaccharide-induced mouse lethality by resveratrol. Food Chem Toxicol 48: 1543-1549, 2010.

17. Murakami M, Yoshihara K, Shimbara S, Lambeau G, Singer A, Gelb MH, Sawada M, Inagaki N, Nagai H and Kudo I: Arachidonate release and eicosanoid generation by group IIE phospholipase $\mathrm{A}(2)$. Biochem Biophys Res Commun 292: 689-696, 2002.

18. Zhou JH, Wei XM, Lan HH, Zhen N, Li JW and Chen $\mathrm{H}$ Protective effects of resveratrol on acute lung injury in septic rats and its effect on HMGB1 and TLR4 mRNA expression. Chin Pharmacol Clin 31: 138-139, 2015.

19. Palomera-Ávalos V, Griñán-Ferré C, Izquierdo V, Camins A, Sanfeliu C, Canudas AM and Pallàs M: Resveratrol modulates response against acute inflammatory stimuli in aged mouse brain. Exp Gerontol 102: 3-11, 2018

20. Kim YA, Kim GY, Park KY and Choi YH: Resveratrol inhibits nitric oxide and prostaglandin E2 production by lipopolysaccharide-activated C6 microglia. J Med Food 10: 218-224, 2007.

21. Lei M, Wang JG, Xiao DM, Fan M, Wang DP, Xiong JY, Chen Y Ding Y and Liu SL: Resveratrol inhibits interleukin 1 $\beta$-mediated inducible nitric oxide synthase expression in articular chondrocytes by activating SIRT1 and thereby suppressing nuclear factor- $\kappa$ B activity. Eur J Pharmacol 674: 73-79, 2012.

22. Oh YC, Kang OH, Choi JG, Chae HS, Lee YS, Brice OO, Jung HJ Hong SH, Lee YM and Kwon DY: Anti-inflammatory effect of resveratrol by inhibition of IL-8 production in LPS-induced THP-1 cells. Am J Chin Med 37: 1203-1214, 2009.

23. Ge L, Liu L, Liu H, Liu S, Xue H, Wang X, Yuan L, Wang Z and Liu D: Resveratrol abrogates lipopolysaccharide-induced depressive-like behavior, neuroinflammatory response, and CREB/BDNF signaling in mice. Eur J Pharmacol 768: 49-57, 2015

24. Ma C, Wang Y, Dong L, Li M and Cai W: Anti-inflammatory effect of resveratrol through the suppression of NF- $\kappa \mathrm{B}$ and JAK/STAT signaling pathways. Acta Biochim Biophys Sin (Shanghai) 47: 207-213,2015.

25. Gualdoni GA, Kovarik JJ, Hofer J, Dose F, Pignitter M, Doberer D Steinberger P, Somoza V, Wolzt M and Zlabinger GJ: Resveratrol enhances TNF- $\alpha$ production in human monocytes upon bacterial stimulation. Biochim Biophys Acta 1840: 95-105, 2014.

26. Bhattarai G, Poudel SB, Kook SH and Lee JC: Resveratrol prevents alveolar bone loss in an experimental rat model of periodontitis. Acta Biomater 29: 398-408, 2016

27. Tamaki N, Cristina Orihuela-Campos R, Inagaki Y, Fukui M Nagata $\mathrm{T}$ and Ito HO: Resveratrol improves oxidative stress and prevents the progression of periodontitis via the activation of the Sirt1/AMPK and the Nrf2/antioxidant defense pathways in a rat periodontitis model. Free Radic Biol Med 75: 222-229, 2014.

28. Bai T, Hu X, Zheng Y, Wang S, Kong J and Cai L: Resveratrol protects against lipopolysaccharide-induced cardiac dysfunction by enhancing SERCA2a activity through promoting the phospholamban oligomerization. Am J Physiol Heart Circ Physiol 311: H1051-H1062, 2016

29. Imamura Y, Yoshikawa N, Murkami Y, Mitani S, Matsumoto N, Matsumoto H, Yamada T, Yamakawa K, Nakagawa J, Ogura H, et al: Effect of histone acetylation on N-Methyl-D-Aspartate 2B receptor subunits and interleukin-1 receptors in association with nociception-related somatosensory cortex dysfunction in a mouse model of sepsis. Shock 45: 660-667, 2016

30. Wang Y, Wang X, Zhang L and Zhang R: Alleviation of acute lung injury in rats with sepsis by resveratrol via the phosphatidylinosito 3 -kinase/nuclear factor-erythroid 2 related factor $2 /$ heme oxygenase-1 (PI3K/Nrf2/HO-1) pathway. Med Sci Monit 24 3604-3611, 2018.

31. Hao E, Lang F, Chen Y, Zhang H, Cong X, Shen X and Su G: Resveratrol alleviates endotoxin-induced myocardial toxicity via the Nrf2 transcription factor. PLoS One 8: e69452, 2013.

32. Wang N, Mao L, Yang L, Zou J, Liu K, Liu M, Zhang H, Xiao X and Wang K: Resveratrol protects against early polymicrobial sepsis-induced acute kidney injury through inhibiting endoplasmic reticulum stress-activated $\mathrm{NF}-\mathrm{\kappa B}$ pathway. Oncotarget 8 : 36449-36461, 2017.

33. Li H: Negative regulation of Keap1-Nrf2 pathway by NF- $\kappa$ B pathway and its molecular mechanism. Tianjin University, Publication no. Q257, 2009

34. Teboul JL and Duranteau J: Alteration of microcirculation in sepsis: A reality but how to go further? Crit Care Med 40 : $1653-1654,2012$
35. Lundy DJ and Trzeciak S: Microcirculatory dysfunction in sepsis. Crit Care Nurs Clin North Am 23: 67-77, 2011.

36. Holthoff JH, Wang Z, Seely KA, Gokden N and Mayeux PR Resveratrol improves renal microcirculation, protects the tubular epithelium, and prolongs survival in a mouse model of sepsis-induced acute kidney injury. Kidney Int 81: 370-378, 2012

37. Pendurthi UR, Meng F, Mackman N and Rao LV: Mechanism of resveratrol-mediated suppression of tissue factor gene expression. Thromb Haemost 87: 155-162, 2002.

38. Hamburger T, Broecker-Preuss M, Hartmann M, Schade FU, de Groot H and Petrat F: Effects of glycine, pyruvate, resveratrol, and nitrite on tissue injury and cytokine response in endotoxemic rats. J Surg Res 183: e7-e21, 2013.

39. Zhang M, Jing LJ and Jing CH: Protective effect of resveratrol on injured organs In endotoxic shock rats. Chin Crit Care Med 7: 352-354, 1995

40. Larrosa M, Azorín-Ortuño M, Yañez-Gascón MJ, GarcíaConesa MT, Tomás-Barberán F and Espín JC: Lack of effect of oral administration of resveratrol in LPS-induced systemic inflammation. Eur J Nutr 50: 673-680, 2011

41. Aydın S, Sahin TT, Bacanlı M, Taner G, Basaran AA, Aydın M and Başaran N: Resveratrol protects sepsis-induced oxidative DNA damage in liver and kidney of rats. Balkan Med J 33: 594-601, 2016.

42. Lou SC: Protective effect and mechanism of resveratrol on cardiac function in septic shock rats. China Medical University, Publication no. AAT 3411606, 2017.

43. Wang X, BuechlerNL, Yoza BK, McCallCE and Vachharajani VT: Resveratrol attenuates microvascular inflammation in sepsis via SIRT-1-Induced modulation of adhesion molecules in ob/ob mice. Obesity (Silver Spring) 23: 1209-1217, 2015.

44. Yang XJ: Protective effect of resveratrol on myocardial injury in septic rats. Chin Pharmacol Clin Med 31: 51-54, 2015.

45. Gan YZ: Protective effects of resveratrol on acute kidney injury in septic rats and its effect on the expression of NF- $\mathrm{kB}$ p65. Southern Medical University, Publication no. R631.2 R285.5, 2016.

46. Huang J: Effects of resveratrol on the expression of inflammatory factors and myocardial damage in sepsis. Guangzhou University of Traditional Chinese Medicine, 2015.

47. Wang Y: The effect of resveratrol on hemorheology of lipopolysaccharide challenged rats. Hebei Northern College, Publication no. R572.2, 2015

48. Gan Y, Tao S, Cao D, Xie H and Zeng Q: Protection of resveratrol on acute kidney injury in septic rats. Hum Exp Toxicol 36 : 1015-1022, 2017

49. Li M: Protective effect and mechanism of resveratrol on acute kidney injury in septic rats. Central South University, Publication no. R459.7 2013.

50. Chen L, Yang S, Zumbrun EE, Guan H, Nagarkatti PS and Nagarkatti M: Resveratrol attenuates lipopolysaccharide-induced acute kidney injury by suppressing inflammation driven by macrophages. Mol Nutr Food Res 59: 853-864, 2015.

51. Bah I, Kumbhare A, Nguyen L, McCall CE and El Gazzar M: IL-10 induces an immune repressor pathway in sepsis by promoting S100A9 nuclear localization and MDSC development. Cell Immunol 332: 32-38, 2018.

52. Park HJ, Lee SJ, Cho J, Gharbi A, Han HD, Kang TH, Kim Y, Lee Y, Park WS, Jung ID, et al: Tamarixetin exhibits anti-inflammatory activity and prevents bacterial sepsis by increasing IL-10 production. J Nat Prod 81: 1435-1443, 2018.

53. Yu YX, Fan J and Zhang M: Protective effects of resveratrol on acute lung injury in septic rats and its effect on the expression of inflammatory protein-2, IL-10 and IL-18 in macrophages. Chin J Biol Prod 25: 1003-1006, 2012

54. Cerqueira AM, Khaper N, Lees SJ and Ulanova M: The antioxidant resveratrol down-regulates inflammation in an in-vitro model of Pseudomonas aeruginosa infection of lung epithelial cells. Can J Physiol Pharmacol 91: 248-255, 2013.

55. Silswal N, Reddy NS, Qureshi AA and Qureshi N: Resveratrol downregulates biomarkers of sepsis via inhibition of proteasome's proteases. Shock 50: 579-588, 2018

56. Li WG and Chen XP: Effect of resveratrol on TNF- $\alpha$ promoter region of THP-1 cells induced by bacterial lipopolysaccharide. Chin J Gen Surg 25: 686-692, 2016

57. Chen GD, Yu WD and Chen XP: sirT1 activator represses the transcription of TNF- $\alpha$ in THP-1 cells of a sepsis model via deacetylation of H4K16. Mol Med Rep 14: 5544-5550, 2016.

58. Sebai H, Ristorcelli E, Sbarra V, Hovsepian S, Fayet G, Aouani E and Lombardo D: Protective effect of resveratrol against LPS-induced extracellular lipoperoxidation in AR42J cells partly via a Myd88-dependent signaling pathway. Arch Biochem Biophys 495: 56-61, 2010. 